\title{
Vocabulaire de Fellag : une innovation lexicale au service d'un humour translinguistique
}

\author{
Moussaouer, Abderahim \\ Laboratoire Lexiques, Dictionnaires, Informatique CNRS-UMR 7187, Université Paris13-Sorbonne Paris Cité
}

a.moussaouer@hotmail.fr

Résumé. Nous visons dans cette étude un but principal, décrire le vocabulaire de Fellag - homme de théâtre, écrivain et comédien algérien qui fait partie de ceux qui fondent leurs sketchs sur le langage et le maniement de la langue - à travers lequel nous constatons une large tendance à la créativité lexicale résultant d'un remarquable contact de langues dans ses spectacles. Cette idée de s'inscrire dans un humour translinguistique est en rapport avec la nature de son public qui est, en majorité, comme lui, c'est-à-dire bilingue ou trilingue. Sa bonne maîtrise de la langue française, entre autres, lui donne la possibilité de créer des mots, détourner des expressions et (dé)former le sens. Cette faculté est l'une des particularités les plus attirantes en ce qui concerne langue de ses spectacles. Cette recherche tente également de souligner le rôle de l'innovation lexicale dans son humour. Ce que nous abordons, là, en termes d'innovation lexicale est basé sur la création par emprunts réciproques, néologismes et hybridation d'où l'intérêt d'en souligner et d'en comprendre les mécanismes de création. Ces trois phénomènes linguistiques ne peuvent être négligés, surtout lorsqu'il s'agit de l'étude d'un discours humoristique où les langues française, berbère et arabe interagissent entre elles, et laissent constater des traces d'une langue dans une autre langue.

\begin{abstract}
This study aims at describing Mohamed Fellag's humoristic discourse. In fact, this paper focuses on his vocabulary, particularly his wide tendency for lexical creativity, which results from a remarkable language contact. This plurilingual aspect of his shows echoes the multicultural background of his audience. His good mastery of French, Arabic and Berber languages gives him the possibility of creating words and meanings and diverting expressions. This linguistic creativity constitutes one of the most attractive aspects of his shows. Moreover, this paper seeks to highlight the significance of lexical innovation in his shows, through a close study of mutual loans, neologisms, hybridisation and their process of creation. These linguistic phenomena are not to be neglected, mainly in Fellag's discourse in which three languages (French, Arabic and Berber) interact and influence each other.
\end{abstract}




\section{Introduction}

"[...] Un mot qui manque en arabe dialectal, hop, on le prend en français et on le conjugue en arabe, on le triture, on en fait un mot [...]» explique Fellag. Tel est le cas de t-gouvern-iw ${ }^{l}$, qui veut dire en arabe dialectal «vous gouvernez », conjugué à l'inaccompli -qui est l'équivalent du présent de l'indicatif en arabe- avec la deuxième personne du pluriel vous. Ce qui attire notre attention dans ce cas est le radical français -gouvern- auquel on a rajouté deux morphèmes grammaticaux : le $t$ - en début (pronom personnel qui correspond à la fois à « tu » et à « vous » en début des verbes conjugués à l'inaccompli); et le -iw (qui correspond à la terminaison «-ez») en terminaison des verbes dont l'infinitif se termine par $-i$, conjugués à l'inaccompli avec la deuxième personne du pluriel vous. Dans la pratique des langues en Algérie, Fellag aurait pu dire aussi : un mot qui manque en français, on le prend en arabe et on le conjugue en français; ou encore : un mot qui manque en berbère, on le prend en français et on le conjugue en berbère. Cela peut s'expliquer par le fait que les langues, souvent en contact, s'échangent des mots ${ }^{2}$ qui, par la suite, épousent les marques phonétiques et morphosyntaxiques des langues cibles.

\section{Le contact de langues comme source d'emprunts}

La pratique langagière dans les spectacles de Fellag est très particulière parce que le comédien n'hésite pas à faire appel aux trois langues (français, arabe et berbère) -qu'il a utilisées précédemment en se produisant sur scène ${ }^{3}$ - même lorsqu'il s'agit d'un spectacle présenté en une seule langue. Autrement dit, l'alternance codique, conçue généralement comme le mélange des langues dans un même énoncé, constitue un des ferments essentiels de la langue du comédien. En effet, il arrive que ce dernier présente un spectacle en français, mais on constate la présence de mots arabes et berbères ayant des fonctions linguistiques, mais aussi sociales et culturelles. Le même phénomène est constaté lorsque le comédien se produit sur scène en arabe ou en berbère : des mots français s'enchâssent dans son discours et épousent la morphologie et la phonétique de ces deux langues.

Si l'on observe cette pratique on remarque une transgression relative du code des trois langues, notamment au niveau du lexique. La transgression dont il est question s'explique, entre autres, par le phénomène d'emprunt lexical défini selon Foudil Cheriguen (1989-2 :55) comme étant une unité de fonctionnement comportant une ou plusieurs parties, toutes susceptibles d'usage syntaxique autonome. Il est mis en morphologie [...] parce que passé dans la langue cible. La base lexicale relève de la langue source. Il a donc un rapport avec celle-ci (rapport étyomogique). 
À l'ombre de cette définition structurée, l'emprunt se présente comme un mot, utilisé par un système linguistique, et qui est importé par un autre système linguistique. Suivant cette logique, Fellag, comme tout autre locuteur algérien, utilise les mots de la langue arabe ou berbère dans le système linguistique français et leur applique pour les circonstances de la communication toutes les ressources de la langue d'accueil notamment les règles de dérivations morphologiques, syntaxiques, lexicologique et sémantique (préfixation / suffixation / composition / adjonction d'actualisateurs et de déterminants, de marque de genre et de nombre...), comme l'indique Yacine Derradji (1999). En revanche, nous avons constaté un apport du français à l'arabe et au berbère dans des spectacles (présentés dans ces deux langueslà) à travers des mots empruntés au français.

\subsection{Verbes formés sur des radicaux empruntés}

Les emprunts peuvent servir de radicaux, pour des formes verbales, auxquels on rajoute des affixes, des pronoms personnels ou des compléments d'objet direct (souvent en arabe et en berbère, le pronom personnel et le COD sont attachés au verbe). Dans ce qui suit, nous avons des verbes formés sur des radicaux empruntés au français, à l'arabe et au berbère.

\subsubsection{Verbes formés sur des radicaux français}

- [...] y en a même un, il est venu avec la bouteille de butagaz, il a dit: "je vais la gazer [...] drouk n-gaz-iha » [...]. (Un bateau pour l'Australie).

$\boldsymbol{n}$-gaz-iha veut dire, en arabe dialectal, « je vais la gazer ».

- [...] ya xo, yâk râkûm t-stérilis-iw lamwâs tâckûm[...] = [...] mon frère, j'espère que vous stérilisez vos rasoirs [...]. (Cocktail Khorotov).

$\boldsymbol{t}$-stérilis-iw, du verbe stériliser, correspond à «vous stérilisez», conjugué en arabe algérien, à l'inaccompli avec la deuxième personne du pluriel.

- [...] yiwan ath-yecras-i u-camyûn [...] = [...] quelqu'un qui se fait écraser par un camion $[\ldots]$. (Cocktail Khorotov).

ath-yecrasi veut dire, en kabyle, « l'écraser » et dans notre exemple « se faire écraser ».

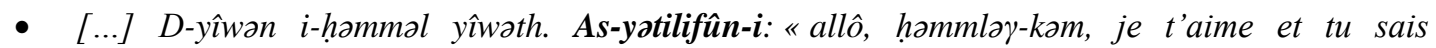
à quel point! fall-âm ad-travers-îy la mer Méditerranée $[\ldots]=[\ldots]$ C'est l'histoire d'un garçon qui aime une fille. Il lui téléphone: "allô, je t'aime, je t'aime et tu sais à quel point! Pour toi je traverserai la mer Méditerranée [...] (Cocktail Khorotov).

as-yatilifûn-i est l'adaptation morphologique et phonétique, en kabyle, de « il lui téléphone ».

ad-travers-îy est l'adaptation morphologique et phonétique, en kabyle, de « je traverserai ».

\subsubsection{Verbes formés sur des radicaux arabes/berbères}

- [...] c'était la seule fille qui était toute seule dans la boite. [...] Je me suis mis comme ça derrière une colonne en marbre, et je l'ai gabrée [...] (Djurdjurassique Bled).

gabrée, participe passé féminin, du verbe gabrer obtenu par l'ajout du suffixe verbal -er au radical arabe gâbor qui veut dire « capter par les yeux »".

- [...] les trois géants sont allés attraper Arezki. Ils l'ont guetté. Ils ont fini par le trouver. Ils l'ont attaché. Ils l'ont hallalisé, merguezé, cachirisé [...] (Un bateau pour l'Australie). 
hallalisé et cachirisé : conjugaison de hallaliser et kachiriser au passé composé. Ces verbes sont obtenus par l'ajout du suffixe -iser au radicaux, à la fois berbères et arabes, hallal («licite» selon le rite musulman) et cachir qui veut dire «saucisson». Notons que le saucisson est appelé cachir en Algérie.

- [...] La fille est allée voir son papa, elle lui a dit : "ô papa! ô tu sais, j’ai rencontré un garçon magnifique et je l'aime, papa!»[...]. "Il s'appelle Mouhh- ». La fille, essoufflée, n'arrive pas à prononcer le [h]. [...] Ils l'ont emmenée à l'hôpital, ils lui ont enlevé le (ح) qui s'est accroché à la gorge. Alors, ils l'ont déhahifiée. Elle est revenue à la maison : «il s'appelle Mo-amed » [...] (Djurdjurassique Bled).

déhahịifiée de dé-hah-ifier. Le préfixe privatif dé- et le suffixe verbal -ifier joints à la base arabe hâ. Le hâ est le nom de la lettre arabe $\tau$.

\subsection{Noms formés sur des radicaux empruntés}

En revanche, les noms aussi peuvent être formés à partir de radicaux empruntés à d'autres langues. Dans ce cas, ils peuvent être l'objet d'une affixation au sein de la langue cible, comme ils peuvent garder leurs formes tels qu'introduits dans la langue source.

\subsubsection{Noms formés sur des radicaux français}

- [...] yiwan ath-yecras-i u-camyûn [...] = [...] quelqu'un qui se fait écraser par un camion [...]. (Cocktail Khorotov).

-camyûn, de acamyûn, veut dire « camion » en kabyle.

- [...] yəkûn el-pârti tâع arrğâl, el-pârti tâع onnsâ, el-parti tâe onnâs allî yəchûfu onnsa ğâyzin $[\ldots]=[\ldots]$ Il y aura le parti des hommes, le parti des femmes, le parti de gens qui regardent les femmes passer [...] (Cocktail Khorotov).

el-pârti : de parti emprunté au français, mis en morphologie avec l'article défini arabe el-.

- [...] Eəndhûm wâhad el-bwâta! Wâhad el-bwâta! Une boite magique! [...] = [...] Ils ont une boite! Ils ont une boite! Une boite magique [...] (Cocktail Khorotov).

el-bwâta : du mot boite emprunté de l'arabe dialectal au français. Le el- du début est un article défini; le $-a$ à la fin est une marque flexionnelle relative aux noms féminins singuliers en arabe dialectal.

- [...] à la fin du match, l'équipe qui gagne, elle boit les deux bouteilles de gazouse [...] (Un bateau pour l'Australie).

gazouse [gazu:z] est l'adaptation du mot gazeuse aux systèmes vocaliques arabe et berbère. En l'absence du son [ø] dans leurs langues, les locuteurs ${ }^{5}$ arabophones et berbérophones prononcent le son [u] (ce dernier fait partie des trois sons sur lesquels est basé les systèmes vocaliques berbère et arabe : [a], [u] et [i]).

\subsubsection{Noms formés sur des radicaux arabes/berbères}

- [...] Les anciens partis commencent à réapparaître, et d'autres sont créés [...] les Choumistes, les Khoubzistes [...] (Cocktail Khorotov).

Choumistes: avec l'ajout du suffixe français -iste au nom (arabe et berbère) chouma qui veut dire "chique», Fellag évoque un parti politique fondé par les amateurs du tabac à chiquer; idem pour le mot Khoubzistes construit sur khoubz, qui veut dire en arabe/berbère "pain", et le suffixe français (d'origine grecque) -iste. Ce dernier est employé pour désigner, de façon générale, les personnes qui gagnent leur pain de façon opportuniste et malhonnête. 
- [...] J'ai fait une heure de gabration intensive. La gabration, pour ceux qui ne parlent pas l'arabe, c'est à peu près la captation avec les yeux [...] oui parce que ces choseslà chez nous, l'amour, les femmes et tout ça, on ne parle jamais de ça [...] (Djurdjurassique Bled).

gabration: mot construit sur la base arabe (voire berbère actuellement) gabar, avec l'ajout du suffixe nominal -ation. Dans ce passage, Fellag n’a pas hésité à donner le sens du mot.

Ces emprunts -ou constructions basées sur des radicaux empruntés- repérés dans les spectacles de Fellag relèvent exclusivement du discours parce qu'il est question, là, du discours humoristique, mais l'objectif de leur apparition dans ce type de discours ne peut être différent de celui de leur usage dans la langue. Dans ce sens Christine Jacquet-Pfau (2003:81) considère que l'emprunt constitue un mode d'enrichissement linguistique commun à toutes les époques et à toutes les langues dès qu'il y a eu contact avec au moins une autre langue. L'emprunt lexical, que nous avons étudié dans le cadre ci-dessus, figure au nombre des principaux moyens qu'a une langue d'enrichir son stock lexical.

\section{Qu'en est-il des xénismes ?}

Dans les spectacles de Fellag, les spectateurs français ou autres qui ne parlent ni arabe, ni berbère, entendent parfois des mots étrangers, crus, qui ne se soumettent pas aux règles de dérivation ou de morphologie française. Ce sont ces mots -noms, verbes ou adjectifs- arabes ou berbères qui sont utilisés (dans un discours en français) sans que leur aspect morphologique et phonétique change. Foudil Cheriguen (1989-2:55) parle de xénisme et le définit comme étant une unité de fonctionnement pouvant comporter une ou plusieurs parties; chacune d'elles étant susceptible d'usage syntaxique autonome. Il n'est pas mis en morphologie parce que n'appartenant pas (ou pas encore) à la langue cible pour laquelle sa base est étrangère.

Les xénismes recueillis dans les textes de Fellag sont en majorité des mots simples et de la catégorie nominale, et les verbes et les adjectifs sont très rares. Nous avons sélectionné des exemples qui relèvent :

- de l'art culinaire: [...] je vais lui mettre les asqaqer de chez nous, là, les épices nucléaires [...] (Djurdjurassique Bled).

osqaqer veut dire « épices » en arabe dialectal.

- $\mathrm{du}$ comportement humain : [...] alors, les filles, quand elles arrivent, elles passent à côté de nous. Nous, zacmâ, c'est la hchuma! eh ben oui, c'est le respect, c'est la pudeur $[\ldots]$ (Un bateau pour l'Australie).

zacmâ : c'est l'équivalent, en arabe et en berbère, de la locution conjonctive « comme quoi ».

h̆chuma est un substantif qui véhicule plusieurs sens selon le contexte: "honte», "pudeur», « respect», « timidité », etc.

- $\mathrm{du}$ style vestimentaire : [...] celle-là tu lui mets un hîŭgâab, mets-là de côté [...] (Djurdjurassique Bled).

ḥ̂̆ğâb: appellation généralisée, en arabe littéraire, en arabe dialectal et même en berbère de « voile ».

- des différentes substances utilisées pour diverses raisons: la chemma (Djurdjurassique Bled) qui signifie en arabe et en berbère «tabac à chiquer»; ihochkûlen et osḥ̂ur (Un bateau pour l'Australie) qui signifient, en berbère et en arabe dialectal respectivement, « philtres » ou « sortilèges ». 
Il convient également de mettre la lumière sur l'expression incâddîn, présente presque dans tous les spectacles de Fellag et utilisée des dizaines de fois. C'est une locution verbale qui, ayant pour objectif d'insulter, signifie «soit maudite la religion de [...]» ou dans son usage simplifié «soit maudit [...]». Elle est suivie de la personne ou de la chose que l'on veut insulter :

- [...] et j'ai pleuré pendant trois jours la mort de Spartacus, en maudissant Jules César incâddin yəmmâ-s [...] (Le dernier chameau).

incâddin yəmmâ-s : en kabyle, « soit maudite sa mère ».

- [...] Il est empoisonné ton truc, je vais attraper la lèpre avec ça, moi! T'es con Mohamed ! [...] Incâddin bâb̂̂a-k ! [...] (Le dernier chameau).

Incâddin bâbâ- $\boldsymbol{k}$ : en arabe dialectal, « soit maudit ton père ».

- [...] Déjà, là, en tant que larve: [...] je vais rester trois milliards d'années pour devenir un berbère! [...] moi, je veux tout de suite. Incâddin Darwin [...] (Djurdjurassique Bled).

Incâddin Darwin : "soit maudit Darwin" (en arabe et en berbère). En signe de précipitation, les larves veulent devenir des berbères très rapidement, sans être obligées d'attendre tant d'année, et sans être obligées d'évoluer d'où l'Evolutionnisme de Darwin.

L'expression incâddin est susceptible d'usage syntaxique autonome dans le sens de «merde» ou «putain» comme dans l'exemple: [...] Chez nous lorsqu'un type plante un clou, quand il arrive au milieu : "incâddin, je m'ennuie » [...] (Djurdjurassique Bled).

Nous avons constaté aussi l'usage de xénismes adjectifs, qu'ils soient arabes ou berbères. Par exemple :

- [...] Enfin, le lion, le seul lion d'Algérie. C'est un lion complètement mčsrčor! Je te jure. Il a au moins cent quatre-vingt-dix ans [...] (Un bateau pour l'Australie).

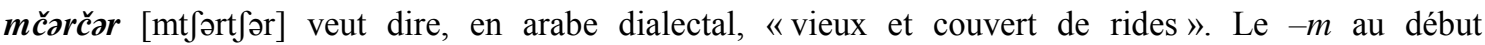
du mot est un préfixe, de l'arabe dialectal, qui accompagne généralement les adjectifs qualificatifs.

- [...] Et moi, j’étais là, à l'entrée, dans un costume tout étriqué, tout serré. C'est un costume complètement mazbôt [...] J'ai mis trois jours pour rentrer dedans, tellement il était serré [...] (Un bateau pour l'Australie).

L'adjectif mazbôt de l'arabe dialectal trouve sa signification dans le passage. En effet, Fellag parle d'un costume « étriqué » et « serré ».

L'usage de ces deux adjectifs mčorčor et məzbôt a pour but d'appuyer la description du lion et du costume en faisant recours à des mots provenant d'une langue partagée entre le comédien et une grande partie de son public. C'est un moyen de provoquer un sentiment ludique parce que, comme nous l'avons constaté, à chaque fois que Fellag introduit des mots ou des expressions en arabe ou en berbère, son public réagit par le rire.

\section{Néologie et hybridation}

Étant donné que l'humour favorise une remarquable indépendance langagière en termes de créativité linguistique et de jeux sur la langue, les spectacles de Fellag sont caractérisés par une large tendance à la néologie. Pour en délimiter le concept, cette dernière peut être traitée sous différentes approches et ne pas avoir forcément une définition consensuelle. Jean-François Sablayrolles (2000:13) indique que la néologie n'est sans doute pas un concept discret, mais 
comporte plutôt différents degrés sur une échelle. La conception large et scalaire de la néologie explique la variabilité des jugements au sujet des néologismes.

Dans le Dictionnaire de linguistique et des sciences du langage, la néologie est définie comme étant " le processus de formation de nouvelles unités lexicales » (Dubois et al., 1994: 334). Ce processus met en évidence un ensemble de procédés permettant la création de nouvelles unités lexicales. Dans le même dictionnaire (p.335) « on appelle néologisme tout mot de création récente ou emprunté depuis peu à une autre langue, toute acception nouvelle d'un mot déjà ancien ". C'est donc le résultat d'une création nouvelle portant sur le plan formel ou un sens nouvellement attribué à un mot déjà existant dans le lexique d'une langue.

L'étude néologique que nous avons envisagé de mener, dans le cadre de cet article, s'articule autour des néologismes que Fellag introduit par hybridation, considérée comme un processus spécifique de créativité néologique ${ }^{6}$. Elle se réalise par l'adjonction d'un élément provenant d'une langue A à un élément provenant d'une langue B. Notre étude de la néologie hybride se propose d'aller au-delà du plan de la morphologie dérivationnelle (basée sur la composition, la composition savante et la dérivation), jusqu'à atteindre la taille des locutions ou expressions, en ayant pour repère la lexie, que Jean-François Sablayrolles qualifie de l'unité la plus appropriée pour l'étude des néologismes, et que Bernard Pottier (1974) définit comme étant l’unité lexicale mémorisée.

\subsection{Hybridation par dérivation}

Considérée habituellement comme un mode de composition, l'hybridation touche ou est aussi un mécanisme qui permet d'obtenir des dérivés, que l'on appelle « dérivés hybrides ». Pour étayer cette idée, nous avons repéré les exemples suivants :

- À partir du mot, à la fois berbère et arabe, hitț, qui veut dire «mur», Fellag met le point sur une pratique quotidienne hittisme, devenue une doctrine, voire une philosophie qui consiste à ce que les jeunes algériens, diplômés ou pas, passent tout leur temps adossés aux murs (à cause du chômage). Ces jeunes chômeurs sont appelés hițistes. Ces deux lexies néologiques affichent leur apparition dans les trois spectacles Cocktail Khorotov, Djurdjurassique Bled et Le dernier chameau.

Hittistes: de hitt, en arabe, qui veut dire «mur»; avec l'ajout du suffixe français (d'origine grecque) -iste qui en fait une doctrine, une pratique sociale.

hitṭisme est obtenu par l'ajout du suffixe -isme au mot hițt.

- [...] Mais, Jésus, tu sais bien que c’est la misère qui m'a amené là! Et toi tu sais ce que c'est la misère! Si, toi, tu me comprends pas, qui va me comprendre? Après tout, toi aussi tu étais un ex-hittiste à Jérusalem [...] (Djurdjurassique Bled).

ex-hittiste est une autre forme de lexie hybride dérivée du mot hitt pour désigner, avec l'ajout du préfixe $e x$-, une personne qui a cessé d'être hịttiste.

- [...] Y a les Français qui vous regardent. Zacmâ c'est vous qui avez fait la guerre, vous avez sorti les Français. Toz! Et retoz [...] (Djurdjurassique Bled).

retoz est obtenu partir du mot arabe (voire berbère) toz, qui signifie littéralement «pet», dans le sens du gaz qui sort par les fesses, mais son sens le plus fréquent est un refus catégorique de façon familière. Fellag introduit un dérivé hybride en rajoutant le préfixe de répétition re-.

Le principe de dérivation hybride s'applique aussi sur des exemples que nous avons cités précédemment, en l'occurrence Choumistes, Khoubzistes, gabration (suffixation nominale); déhahifier, hallaliser et cachiriser (suffixation verbale). 


\subsection{Hybridation par composition}

Le même mot hițt donne lieu à des composés hybrides :

- [...] mais Mohamed, c'était l'un des membres fondateurs du Hitṭisme. Il était docteur en Hittologie [...] (Djurdjurassique Bled).

Le comédien va loin dans sa créativité et crée -avec l'ajout du suffixe -logie qui signifie science ou étude de quelque chose- une science qui s'appelle Hittologie. C'est l'étude du phénomène de Hițtisme.

- $\quad[. .$.$] il était numéro un au Hitț-parade { }^{7}$ [...] (Djurdjurassique Bled).

On parle d'habitude de Hit-parade qui est un classement établi selon la popularité d'une chanson, d'un film, etc. Mais, là, Fellag évoque un classement établi selon la popularité des hittistes.

- [...] à partir d'aujourd'hui, pour tous vos problèmes de santé, un seul docteur, le docteur Flouche! Voilà! Docteur en Khouzzanologie intercostale [...] (Cocktail Khorotov).

Avec l'ajout du suffixe -logie au mot arabe khouzzan qui veut dire «pincement» (douleur), le comédien se présente en tant que spécialiste en khouzzanologie, cette dernière consiste à soigner les douleurs intercostales par le rire.

- [...] il n'y avait rien, c'était le néant, c'était le grand-walou. Et puis, un jour, il y a eu le fameux Bigbang [...] (Djurdjurassique Bled)

Dans l'ordre adjectif + nom le nom arabe et berbère walou, qui signifie l'absence totale de quelque chose ou «rien du tout», est joint à l'adjectif français grand. Par grand-walou Fellag veut dire «néant ».

Les exemples que nous venons de citer peuvent être à la fois des hybrides dénominatifs et des hybrides d'auteur (Jan Kortas, 2009: 533-550). D'abord la présence de certains d'entre eux, dans les spectacles de Fellag, explique que leur usage est là pour dénommer des concepts nouveaux. Ces mots sont utilisés dans les domaines scientifique, politique et social (à travers les contextes dans lesquels ils sont créés). En revanche, on peut également parler d'hybrides d'auteur $\mathrm{du}$ fait que leur caractère assume des fonctions poétiques, ludiques et de dérision, comme est le cas de l'humour de Fellag.

\subsection{Hybridation et locutions}

Nous signalons quelques cas d'hybridation qui touchent les expressions et les locutions nominales et adjectivales.

- Expression: I aime you (Cocktail Khorotov).

De l'expression anglaise I love you qui veut dire « je t'aime ».

- Locution nominale: La Case de l'oncle Moumouh (Djurdjurassique Bled).

Du titre de roman La Case de l'oncle Tom -de l'écrivaine américaine Harriet Beecher Stoween remplaçant le prénom Tom par Moumouh qui est un diminutif de Mohamed.

- Locution nominale: [...] neuf virgule cinq sur l'Échelle n'Rechta [...] (Un bateau pour l'Australie - version berbère).

En remplaçant Richter [rifter] par le nom d'un plat traditionnel berbère Rechta [RəJta]. Ce dernier est, pour le comédien, un moyen de faire un jeu de mots, mais aussi une façon de 
marquer sa culture identitaire. Nous notons que la préposition berbère $n$ qui lie les deux mots de la synapsie correspond à la préposition $d e$.

- Locution adjectivale : à couper au boussâadi (Le dernier chameau).

De la locution figée à couper au couteau qui se dit des choses plus consistantes qu'elles ne devraient l'être. Fellag emploie, à la place de couteau, le nom d'un couteau de fabrication algérienne, spécifique à la région de Boussâada, en l'occurrence le boussâadi.

\subsection{Hybridation et noms propres}

Le phénomène d'hybridation, ou de métissage linguistique, dans les spectacles de Fellag, ne concerne pas seulement les noms communs, il touche aussi les noms propres, notamment les patronymes et les toponymes. Si ce phénomène, dans son discours a lieu, c'est en grande partie grâce aux anecdotes et aux histoires racontées et qui évoquent une certaine interculturalité mettant en jeu des espaces géographiques avec diverses cultures et différentes nationalités. Nous citons quelques exemples :

\subsubsection{Patronymes}

- [...] Le seul lion d'Algérie [...] Il a au moins cent quatre-vingt-dix ans. Je crois que c'est le Maréchal Abdelkader qui l'a offert à l'Émir Bugeaud. Toutes les générations algériennes le connaissent [...] (Un bateau pour l'Australie).

L'attribution, à Abdelkader, du grade de Maréchal qui devrait être attribué à Bugeaud; et l'attribution du grade d'Émir à Bugeaud, ce qui est en revanche propre à Abdelkader. Sachant que les deux personnages L'émir Abdelkader et le Maréchal Bugeaud ont marqué la période d'histoire durant laquelle l'Algérie a été colonisée par la France.

- [...] Le grand psychologue algérien, Si-Muhand Freud, il a dit: "si tu rêves de jambon, ça veut dire, c'est un désir qui est refoulé » [...] (Un bateau pour l'Australie).

Il y a, là, un rapport de ressemblance phonétique entre le prénom de Freud, en l'occurrence Sigmund [sigmund], et un prénom kabyle Si-Muhand [si: muzənd] (avec un [h] aspiré, représenté par [₹] en arabe), d'où la substitution de Si-Muhand à Sigmund.

- [...] En passant dans une ruelle du quartier, le regard d'Arezki a été attiré par un très vieux livre recouvert d'une peau de chèvre, posé sur l'étalage d'un bouquiniste [...] il a pris le livre, il l'a ouvert: L'évangile selon Saint Matthieu. El-Inğ̂il n'sidnâa Mathyû [...] (Un bateau pour l'Australie).

Le disciple de Jésus Saint Matthieu, ou Matthieu-Lévi, se voit transformé en Sidnâ Mathŷu. Cette innovation est motivée par la substitution de sidnâ (qui veut dire «saint» ou «notre seigneur » en arabe) à saint.

\subsubsection{Toponymes}

- [...] Ils m'ont dit: «James Barimor is not here, il n'est pas là, il est à Lalla-bama » [...] (Cocktail Khorotov).

Faisant allusion à l'État d'Alabama qui se situe au Sud-est des États-Unis d'Amérique, le comédien tente un mot-valise hybride avec le mot berbère lalla (qui veut dire «princesse » et qui est aussi une partie du nom de lieu Lalla-Khedidja qui se trouve sur les hauteurs du Djurdjura) et Alabama. Ce choix est motivé par la ressemblance phonétique entre le premier [lala] et les deux premières syllabes du deuxième [ala]. 
- [...] Quand je suis allé à Lalla-bama, on m’a dit que James Barimor était à OuedRhiou De Janeiro [...] (Cocktail Khorotov).

Terme provenant de Rio De Janeiro. Comme le formant Rio [rjo] ressemble phonétiquement à la partie Rhiou [riju] (d'Oued-Rhiou, la deuxième ville du département de Rélizane, en Algérie), Fellag trouve un moyen de faire une fusion hybride amusante, contenant un toponyme arabe Oued-Rhiou et la partie De Janeiro d'un toponyme portugais.

- [...] Quand je suis arrivé on m'a dit qu'il était à New-Delhi-Ibrahim [...] (Cocktail khorotov).

Le toponyme hybride New-Delhi-Ibrahim est le résultat de la jonction du nom de la capitale de l'Inde New-Delhi à celui d'une ville à Alger, en l'occurrence Deli-Ibrahim. Dans ce cas, le trait phonique commun entre les deux toponymes est exprimé par la partie -Delhi [deli] de NewDelhi et Deli [dEli] de Deli-Ibrahim.

- [...] Et à ce moment là, de temps en temps, vous allez voir au hasard, comme ça, Maurice sur son chameau. Il vient de l'oasis de Sidi Germain des prés [...] (Djurdjurassique Bled).

Le comédien trouve une formule amusante et significative. Il remplace Saint par le mot, à la fois berbère et arabe, Sidi qui signifie la même chose : personne qui fait, après sa mort, l'objet d'un culte public et universel. Cette création témoigne de la volonté de Fellag de souligner un aspect religieux algérien à travers un aspect religieux français.

- [ [...] Et il va faire son souk à Bâb el Montreuil [...] (Djurdjurassique Bled).

C'est la création d'un toponyme en provenance de Porte de Montreuil. La lexie française porte est remplacée par la lexie bâb (qui veut dire "porte» en arabe). Dans le spectacle, suivant le contexte, Fellag explique que l'intégration des Algériens en France est aussi facile que la création de toponymes hybrides.

\section{Constructions lexicales multiples à partir d'un radical}

Dans cette partie, nous avons tenté de faire le point sur un aspect lexical, spécifique au discours humoristique de Fellag, qui ne résulte pas forcément d'un métissage linguistique ou du contact des langues. La visualisation des différents sketchs nous a permis de constater que Fellag fait parfois une série de créations à partir d'un mot. En même temps, comme le souligne Dominique Caubet (1998:356), à partir d'une série d'expressions contenant [un mot quelconque], il effectue des permutations, ce qui a pour conséquence de les défiger. Dans ce sens, il est important de souligner qu'un radical peut être la source de : dérivation, composition, synapsies, séquences figées, etc. et peut être également polysémique. Pour cette étude les exemples que nous avons sélectionnés ne sont pas spécialement des mots hybrides ou métissés parce que l'objectif est de voir à quel degré le comédien peut varier dans sa créativité, dans différents contextes, à partir d'une seule unité lexicale.

\subsection{Un mot comme source de dérivation et de composition}

- Le verbe caler donne une série de mots (voire néologismes), comme dans l'exemple : [...] il prenait garde à ce qu'elle ne se fît pas coincer par un caleur professionnel. Vous savez, les caleurs, c'est ces types qui, chez nous, passent des heures entières de journées dans les bus, repèrent les jeunes filles seules, et ils se collent à elles. Ils les calent [...] Il arrive parfois, comme ça, tous d'un coup que, dans un bus, il n'y ait que des caleurs. Tout d'un coup y a soixante-dix caleurs et aucun objet de calage [...] Et le 
type, il espère, il attend, il espère et tout d'un coup, hop, c'est lui qui se fait caler. C'est le caleur-calé [...] le caleur qui cale le caleur qui cale le caleur, jusqu'au chauffeur d'autobus qui, lui aussi, euuh il est calé: Et ben voilà j'ai calé incâddin! [...] (Le dernier chameau).

Fellag nous explique, dans ce passage, le sens de caler et introduit des unités lexicales en sa provenance: caleur (dérivation avec l'ajout du suffixe-eur); caleur professionnel et caleurcalé (composition dans l'ordre $\mathrm{N}+\mathrm{Adj}$ ); objet de calage (synapsie); calé (participe passé du verbe caler). Ce phénomène est une réalité algérienne, déjà ancienne, qui n'est pas tout à fait connue des Français hexagonaux.

- [...] Elle lui glisse le dossier, elle le lui redonne: "Monsieur, nous sommes désolés, mais votre demande de visa est refusée ».

- Madame la France, je ne comprends pas du tout ce refusement [...] tu penses le contraire de la chose tel qu'il est écrit avec le stylo sur la page [...] Pourquoi cette refusation? Pourquoi ce refusage ? [...] (Djurdjurassique Bled).

Le nom refus est, dans ce cas, la source d'une série de néologismes obtenus par dérivation avec l'ajout respectif des trois suffixes nominaux -ement, -ation et -age. Le comédien enchaîne des créations amusantes et ludiques, en réponse à la réaction de son public par le rire pour chaque lexie néologique introduite. Durant ces quelques secondes du spectacle, le comédien se met en interaction avec le public et établit avec lui une sorte de boomerang humoristique, entre les néologismes produits et leur effet ludique constaté chez le public. Dans le passage ci-dessus, Fellag imite le pédantisme d'un personnage qui croit avoir une bonne maîtrise de la langue française, mais qui la « torture » en réalité.

- L'adjectif hallal a servi de base pour certaines lexies néologiques. Nous avons un cas de dérivation hybride dans: [...] Si les Français d'Algérie avaient mis un peu de lben $^{8}$ dans leur vin, on aurait hallalisé nos rapports [...] (Comment réussir un bon petit couscous); un détournement dans: [...] Nous les algériens, comme on ne peut pas boire d'alcool, le baba au rhum, c'est avec de la limonade gazouse. C'est du baba au rhum hallal [...] (Le dernier chameau).

\subsection{Un mot pour une série d'expressions figées}

- Le comédien fait parfois des traductions en chaîne, et à un moment donné de cet enchaînement, on ne retrouve plus le mot -résultat de traduction- qu'on avait au départ. Nous avons pris l'exemple de rîh > «air » et rîh > "vent»: [...] ybîcu même ar-rîh f-

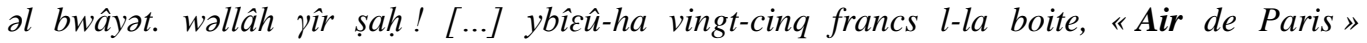
[...] Hnâa on ne produit que du vent. Mcammra blâdna bar-rîh [...] = [...] Ils vendent même $d u$ vent en boites, je vous jure que c'est vrai! [...] Ils le vendent à vingt-cinq francs la boite, "Air de Paris" [...] Nous, on ne produit que du vent. Le vent est abondant dans notre pays [...] (Cocktail Khorotov).

- À partir d'une expression qui contient le mot crise il procède à la permutation, comme dans l'exemple suivant: [...] dârat l-u une crise mystique, kîma hnna cand-na la crise du logement, hûma cand-hum les crises mystiques $[\ldots]=[\ldots]$ Elle lui a fait une crise mystique, comme, nous, nous avons la crise du logement, eux, ils ont des crises mystiques [...] (Cocktail Khorotov).

- Idem pour le mot point contenu dans un certain nombre d'expressions figées. Fellag nous fait assister à un jeu sur les expressions figées: [...] Dernièrement, dans une école, ici, à El-Biar, on raconte qu'un directeur d'école a cassé un élève de 10ans, 
pourquoi? Parce qu'il n'a pas mis deux points sur le ta marbouta ${ }^{9}$ [...] Mais le directeur d'école, il représente le système, et le système, qu'est-ce qu'il veut? Un ta marbouta est un ta marbouta, et il prend deux points au dessus! alors, qu'est-ce qu'il a fait? Il lui a mis les points sur les « $\boldsymbol{i} »$ ! (d'un geste Fellag explique que le directeur d'école donne une raclée à l'élève) mais, c'est des points de suture! [...] (Cocktail khorotov).

Le passage tourne autour du mot point: le ta marbouta avec ses deux points; et les deux expressions figées mettre les points sur les « $i$ » et les points de suture.

- Une série d'expressions qui contiennent l'adjectif unique: [...] Chez nous, pendant trente ans, on a eu un régime unique, un parti unique, un président unique, une pensée unique, mais on avait également un jus de fruits unique. Sur le marché, il n'y avait que du jus d'orange [...] (Le dernier chameau).

Pour parler de l'existence, à une certaine époque, d'un seul jus de fruits, le comédien compare son unicité au régime unique qui gouverne l'Algérie depuis son indépendance. Par le procédé de détournement il évoque un jus de fruits unique comme un clin d'œil pour affirmer sa position en tant que comédien engagé et défenseur de la démocratie.

\subsection{Un mot polysémique}

- [...] Son premier jour à l'armée, le sergent-chef lui a demandé: "Qu'est-ce que tu fais, toi ?». Il lui a répondu: "je suis ingénieur des barrages». Le sergent-chef: " eh ben, toi, ta place est dans les barrages de police ou de l'armée sur les routes. Puisque t'es spécialisé dans les barrages, tu as trouvé ton poste» [...] À la fin de son service national, il a eu peur des faux barrages et il s'est barré d'Algérie [...] (Djurdjurassic Park).

Nous assistons à une série de signifiés que l'on peut attribuer au mot barrage. Le personnage dans ce passage est un ingénieur des barrages, c'est-à-dire des ouvrages barrant des cours d'eau pour faire une retenue. Le sergent-chef le place dans les barrages de police sur les routes. Après avoir accompli son service national, pour échapper aux faux barrages ou à ces obstacles mis en place par des groupes terroristes ou des bandits (et qui donnent l'impression que ce sont ceux de la police), il s'est barré, il a «quitté » le pays. Nous notons que ce dernier cas est un signifié manifesté par le verbe se barrer.

- Entre vivre et travailler en noir (en situation irrégulière), dans le noir (obscurité) et se livrer à des idées pessimistes et mélancoliques (en broyant du noir), le comédien souligne, encore une fois, un mot dont les signifiés sont utilisés dans plusieurs contextes: [...] Il travaillait dans une cave, dans un atelier clandestin, en noir, dans le noir, en broyant du noir. Il mangeait là, il dormait là, il ne sortait jamais [...] (Cocktail Khorotov).

\section{Conclusion}

L'innovation lexicale par emprunt et hybridation et le recours à une stratégie mettant la polysémie en jeu sont très utilisés dans le comique de Fellag. Le contact des langues dans les spectacles de ce dernier joue un rôle principal, dans le sens où cela lui permet d'introduire des emprunts, des xénismes et des unités lexicales hybrides. Mis à part sa volonté d'afficher son appartenance à une communauté socioculturelle maghrébine, le comédien amène des jeux du langage translinguistiques qui, parfois, exigent une connaissance de plusieurs langues. 
La créativité lexicale dans les textes de Fellag vise à mettre en évidence la dénomination de certaines réalités sociales, culturelles et politiques qui constituent le vécu -parfois miséreux et spontané- de ses compatriotes. La fameuse philosophie algérienne le Hittisme est un très bon exemple que l'on peut assigner à une jeunesse pleine d'espoir, mais qui passe des journées entières le corps adossé au mur. Cette situation dramatique n'empêche pas le comédien d'appuyer sa devise qui consiste à « rire de tout ».

Lorsque Fellag recourt à la dérivation hybride, traduit des expressions ou introduit des xénismes et des emprunts, cela veut dire qu'il met en évidence un commun langagier qui le lie aux spectateurs maghrébins ou bilingues, mais aussi une façon d'expliquer que la langue, avec toutes ses richesses, permet de briser toutes ces frontières qui séparent les gens, de diverses cultures et de différentes origines. Pour lui, on peut tout simplement hallaliser au lieu rendre hallal; un Algérien peut lire El-Inğîl n'sidnâ Mathŷ̂ au lieu de feuilleter l'Évangile selon Saint Matthieu et, pour conclure, un musulman, coincé entre sa tradition qui lui interdit de boire l'alcool et une délicieuse pâtisserie que son voisin chrétien prépare, peut manger des babas au rhum hallal arrosés à la gazouse.

\section{Références bibliographiques}

Caubet, D. (1998). Humour et défigement des expressions figées au Maghreb chez les humoristes Mohamed Fellag et Gad Elmaleh. Rencontres Linguistiques Méditerranéennes : Le figement lexical, numéro 1, pp.351-360.

Caubet, D. (2004). Les mots du bled: création contemporaine en langues maternelles, les artistes ont la parole. Paris : L'Harmattan.

Cheriguen, F. (1989). Typologie des procédés de formation du lexique. Cahiers de lexicologie, numéro 55, pp.53-59.

Cortas, J. (2009). Les hybrides lexicaux en français contemporain : délimitation du concept. Meta : journal des traducteurs, numéro 54, pp. 533-550.

Derradji Y. (1999). Le français en Algérie : langue emprunteuse et empruntée. Le français en Afrique, numéro 13 , pp. 71-82.

Dictionnaire Le Nouveau Petit Robert 2010. Paris : Dictionnaires Le Robert-Sejer, cop.2009.

Dubois, J. et al. (1994). Dictionnaire de linguistique et des sciences du langage. Paris : Larousse.

Fellag, M. (1999). Djurdjurassic Bled: Textes de scène. Paris : J-C Lattès.

Jacquet-Pfau, C. (2003) Du statut de l'emprunt en traitement automatique des langues. L'innovation lexicale (éd. J.-F. Sablayrolles). Collection Lexica mots et dictionnaires, pp.79-97.

Moussaouer, A. (à paraître). Néologismes polylexicaux et défigement dans l'humour de Fellag : de la créativité à l'interprétation. Étude de Linguistique Appliquée.

Pottier, B. (1974). Linguistique Générale. Paris : Klincksieck.

Rey, A. et Chantreau, S. (1993). Dictionnaire des expressions et locutions. Paris : Dictionnaires Le Robert.

Sablayrolles, J.-F. (1996-1997). Néologismes : une typologie des typologies. Paris-7 : Cahiers du C.I.E.L, pp.11-48.

Sablayrolles, J.-F. (2000). La Néologie en français contemporain. Paris : Honoré Champion.

Sablayrolles, J.-F. (2012). Des néologismes par détournement ? ou Plaidoyer pour la reconnaissance du détournement parmi les matrices lexicogéniques. JULLION Marie-Christine, LONDEI Danielle, PUCCINI Paola. Recherches, didactiques, politiques linguistiques : perspectives pour l'enseignement du français en Italie, Oct 2009, Milan, France. Francoangeli, pp.17-28, Il punto. <halshs-00735933>. 


\section{Vidéographie/sitographie}

Cocktail Khorotov. (1989), Petit Théâtre de Riadh-el-Fath, Alger. Disponible en ligne, URL: https://www.youtube.com/watch?v=jQqH9HgTN7s

Un bateau pour l'Australie. (1991), Théâtre de Saint-Quentin-en-Yvelines Scène Nationale. Disponible en ligne, URL : https://www.youtube.com/watch?v=v-FBz8 cD8g

Djurdjurassic Park. (1995), Théâtre du gymnase Marie-Bell, Paris. Disponible en ligne, URL : https://www.youtube.com/watch?v=71vNDD5Mp_M

Djurdjurassique Bled. (1998), Théâtre des Bouffes du Nord, Paris. Disponible en ligne, URL : https://www.youtube.com/watch?v=bFLfJLo3K7U

Comment réussir un bon petit couscous. (2003), BRTV. Disponible en 4 parties (enchainement de vidéos), en ligne, URL : https://www.youtube.com/watch?v=30bscMCh94c

Le dernier chameau. (2004), Théâtre des Bouffes du Nord, Paris. Disponible en ligne, URL: https://www.youtube.com/watch?v=VHKfqBTqB6o

\footnotetext{
${ }^{1}$ t-gouvern-iw : l'équivalent, en arabe dialectal maghrébin, de « vous gouvernez ».

${ }^{2}$ Avec le phénomène d'emprunts, une langue $\mathrm{A}$ emprunte des mots à une langue $\mathrm{B}$, et vice-versa.

${ }^{3}$ Il a présenté Djurdjurassique Bled en arabe algérien, Un bateau pour l'Australie en kabyle.

${ }^{4}$ Sens du verbe gâbər selon Fellag, mais aussi celui de son usage habituel.
}

${ }^{5}$ Nous évoquons exclusivement les vieilles personnes qui n'ont peut être pas eu la chance d'aller à l'école ou qui n'ont pas reçu de cours de français. Ce sont les gens qui, à une certaine époque, étaient sous l'emprise de la pauvreté et du colonialisme.

${ }^{6}$ Le procédé d'hybridation est l'une des matrices lexicogéniques (faisant partie des matrices morphosémantiques matrices internes) qui constituent une typologie de néologismes proposée par Jean-François Sablayrolles (1996-97), suite aux travaux de Jean Tournier menés sur la productivité lexicale. Ce dernier a développé une typologie de procédés qu'il a nommés «matrices lexicogéniques", repris et remaniés par Sablayrolles par quelques ajouts ou distinctions supplémentaires.

${ }^{7}$ Cette unité lexicale obtenue par hybridation est aussi un détournement -traitement des cas de défigement par une matrice lexicogénique [...] Ceci nous renvoie directement aux différentes formes de la lexie (mot) parmi lesquelles on trouve les séquences figées, les expressions, les dictons, etc. (Sablayrolles, 2012 :20)- du mot Hit-parade.

${ }^{8}$ Lben veut dire, en arabe, « lait fermenté » ou « petit lait».

${ }^{9}$ Le $t a$, en arabe $\ddot{\partial}$, veut dire $« \mathrm{t} »$. Il apparait en final de mot pour marquer le féminin, mais aussi le nom d'unité des collectifs. Il s'écrit avec deux points suscrits. On le qualifie de marbouta (liée) parce qu'il s'écrit lié (ä) en fin du mot. On a dit liée, au féminin, parce que le ta est féminin. 